\title{
Cypern und seine Flora.
}

\section{Reiseskizze von Paul Sintenis. \\ (Fortsetzung.)}

\section{Panaia tu Kantara.}

Das nächtlicher Weile nicht unter den angenehmsten Umständen erreichte Kloster präsentirte sich im Glanze der Morgensonne als völlig verwilderter aber höchst romantischer Ort. Es liegt auf einem kleinen, nach Süden zu abfallenden Plateau, fast auf dem Rücken des Gebirges, gegen 2000' hoch. Ein kapellenartiges und massiv gebautes, weiss getünchtes Kirchlein steht hart am Südrande des Hochplanes und grenzt in dieser Richtung den Klosterbof ab, dessen übrige Seiten fast durchweg von niedrigen, ganz verfallenen Gebäuden umgeben sind. Auch das lange, düstere Haus, an dessen einem Ende der greise Priester im fensterlosen, schwarzgeräucherten Gemache hauste, dessen anderes Ende uns nothdürftiges Obdach gewährte, und welches fast die ganze Westseite des Hofes bildet, neigt sich nach verschiedenen Richtungen der Windrose, ein Bild der traurigsten Verwahrlosung. Der Hofraum ist mit hohen Kräutern so dicht und üppig bewachsen, dass von dem angehäuften Schutt und Steingetrümmer nicht viel zu sehen ist. Malva parviflora, Chrysanthemum coronarium, Erodium moschatum, E. malacoides und ähnliche bilden die Ruderalflora. Die grossen goldgelben Wucherblumen auf dem saftig grünen Grunde verleihen diesem Orte einen unvergleichlich prächtigen Schmurk.

Rings um das Kloster und hinab an den sanfteren Hängen breiten sich schöne Matten; alte verwahrloste Culturbäume stehen einzeln auf ihnen umher. Keine pflegende Menschenhand hat seit wer weiss wie langer Zeit diese greisenhaften Aprikosen-, Pflaumen-, Mandel- und Delbäume von ihren dürren Aesten befreit; gewissenlos und lüderlich nur übt zuweilen wie es scheint, der Sturm hier oben das Gärtneramt; trotz alledem schmücken sich die Genannten, so viel sie nur können, mit jungen Blättern und schimmernden Blüthen.

Unzählige bunte Blumen durchwirken das saftige Mattengrüu; namentlich häufig sind: Ranunculus lomatocarpus, Adonis dentata, beide vielstengelig, ausgebreitet niederliegend; Trifolium resupinatum und $T r$. tomentosum in enormer Ueppigkeit, kaum ein anderes Pflänzchen neben sich duldend, färben manche Stellen mit hellem Purpur; einige Medicago-Arten treten in ähnlicher Geschlossenheit auf. Nur der einzeln stehende Ranunculus Asiaticus trägt seine an Schönheit Alles überstrahlenden, blutrothen Kronen wie in stolzem Selbstbewusstsein hoch über der niedrigen Blüthenwelt. Eng an das alte Klostergemäuer aber schmiegt sich die goldgelbe Blüthenfülle hochwuchernder Senfstauden (Sinapis alba).

Bald hinter dem Kloster erhebt sich der felsige Bergrücken um noch 200-300' höher; der steile Abhang ist wechselweise mit Cypressengebüsch, Salbei- und Cistensträuchern bewachsen; die letz- 
teren haben hier bereits die ganze Pracht ihrer Blumen entfaltet. Ein paar hundert Schritte gegen Ost zieht sich über der blumigen Matte ein dunkler Cypressenhain am Berghange hinauf; alte Bäume von herrlichem Wuchse. Einige gigantische Eichen (Quercus inermis?) mit weitragenden Aesten, in lachend grünem Schmuck des sich entfaltenden Laubes, stehen am unteren Saume des Wäldchens; - mit leisem Gemurmel rinnt ein krystallklares Bächlein vorüber, welches, im Schatten umwaldeter Felsen in nächster Nähe geboren, eiligen Laufes durch die grüne Dämmerung: dem sonnigen Wiesenplane zustrebt. Lythrum Graefferi verwandelt seine Ufer zu hellrothen Blumenguirlanden, hohe, blühende Doldenpflanzen (Smyrnium Olusatrum) mit glänzend grünem Laube beugen sich schattend über seinen kleinen Spiegel. Süsser Wohlgeruch durchströmt die kleine Waldhalle; den humusreichen Boden bedeckt ein entzückender, dichtgewirkter Blumenteppich von Cyclamen latifolium! Rings um die umfangreichen Eichenstämme schmücken das hier mit einer Sehicht modernden Laubes bedeckte Erdreich, dicht geschaart, in inniger Vereinigung: Trifolium clypeatum, Trif. spiumosum, Vicia gracilis, Lotus creticus, Lagoseris bifida, Crepis Sieberi, Rodigia commutata. Sowohl dieses Wäldchen, als auch die das Kloster umgebenden Obstbäume sind reichbevölkert von befiederten Sängern; hier zwitschert und singt es, namentlich in den Morgen- und Abendstunden aus allen Zweigen und traulich tönt in das helle Lied der Blattmönche, Finken und Grasmücken das Gurren der Wildtauben durchs blumige Revier.

Die Aussicht von Kantara herab nach Süd, Ost und West ist unbeschreiblich grossartig. Den bewaldeten Gebirgsstock hinunter, über die vielen Bergrücken hinweg erschaut das Auge mit Einem Blick die ganze östliche Messorea gleich einer aufgerollten Riesenlandkarte, bis hinab zum Capo Greco, ja bis bis zum Cap Pyla soger, wenn die Luft ganz klar ist, und hinaus ins Unendliche spannt sich das glänzende Meer, welches hier den grossen Golf von Famagosta bildet.

In der engen Klosterzelle richteten wir uns ein, so gut es gehen wollte; umgeben von so erhabener Naturschönheit, wurde mancher Uebelstand völlig übersehen, der sonst geradezu hingereicht hätte, den Aufenthalt völlig zu verleiden. Mein Begriff von einem griechischen Kloster, mit dem ich mindestens einen wohlbestellten Hühnerhof und stattliche Ziegenheerden verknüpfte, erlitt hior gewaltige Einbusse. Der lebensmüde Eremit schien keine irdischen Ansprüche zu kennen; mutterseelenallein hauste er hier oben, das kleine Hündchen war einziger Wächter, Freund und Gefährte aller Schicksale dieses absonderlichen Menschen; nur zufällig war jener Grieche im Kloster über Nacht gewesen, der uns bei unserer Ankunfi Einlass gewährt hatte. Um das ehrwürdige, schöne Haupt des Greises wallten weisse Locken, und ein langer wilder Bart floss von Kinn und Wangen auf das überaus schäbige Priestergewand nieder, welches der Alte Tag und Nacht, jahraus, jahrein auf dem Leibe zu tragen schien. Seine Nahrung bestand allem Anscheine nach zumeist aus 
dem Unkraute des Klosterhofes, d. h. aus den Blättern der Malve; etwas Anderes sah ich ihn nie in seinem schmutzigen Kessel kochen. Corianderblätter und Zwiobel mochten wohl die einzige Würze sein, und was er hiezu als Brot genoss, sah verwittertem Granit wahrhaftig nicht unähnlich. Man darf jedoch hierbei nicht vergessen, dass unser Aufenthalt auf Kantara in die griechischen Fasten fiel.

Die nächsten Tage nach unserer Ankunft widmeten wir der botanischen Durchsuchung der nahen Umgebung des Klosters. Die steinigen Berghänge sind, wie erwähnt, mit Gebüsch bedeckt, welches aus Cupressus horizontalis, Rhamnus Heldreichii, Salvia Libanotica, Cistus Creticus, C. salviaefolius, C. parviflorus und Calycotome villosa besteht. $\mathrm{Zu}$ diesen gesellen sich massenhaft die niedrigeren Sträucher von Thymelaea Tartonraira, Lithospermum hispidulum, Origanum microphyllum, Asparagus horridus, Helichrysum siculum, Phagnalon rupestre, Poterium spinosum. Unter dem oft dicht verwachsenen Gestrüpp sammelten wir: Polygala venulosa, $P$. monspeliaca, Valeriana Dioscoridis, Ziziphora capitata, Micromeria juliana, Linum strictum, Helianthemum apenninum, Hel. arabicum, H. glutinosum, Centaurea Crupinastrum, Atractylis cancellata, Filago prostrata, $F$. germanica, F. spathulata, Micropus bombycinus, Scaligeria Cretica, Lagoecia cuminoides, Ononis sicula, O. breviflora, $O$. reclinata, $O$. ornithopodioides, Medicago coronata, M. circinata, Trifolium Cherleri, Astragalus cyprius, Lotus edulis, Melica sasatilis, M. minuta, Crucianella angustifolia u. a.; selten, an folsigen Stellen stand Asphodelus liburnicus, erst in Knospen; Asph, ramosus blühte noch und war auch hier sehr häufig. Der Rücken des Gebirges, ein langes, nach Norden sanft ansteigendes Plateau, vorherrschend mit silbergrauer Thymelaea Tartonraira bewachsen; bot hier meist einen trallrigen Anblick durch die vielen, wie dürre Besen umherstehenden Meerstrandskiefern. Es ist mir nicht klar geworden, welche Ursachen das Zugrundegehen der schönen Bäume herbeigeführt hatten, ich konnte weder Spuren der Axt noch des Feuers an ihnen gewahren. Häufig zeigten sich alte, sehr grosse und kunstvoll gesponnene Raupennester an den verdorrten Aesten. Hohe, düstere Cypressen, namentlich am Nordsaume des Plateaus um wilde Felspartien geschaart, erhöhen den melancholischen Eindruck, den die spärlich über die Fläche zerstreuten, weissblumigen Cistenbüsche nur wenig mildern. Wir fanden hier oben: Carex panicea, C. gynobasis, Trifolium nigrescens (in grösseren Rasen), Trif. scabrum, Lotus ornithopodioides, Bellis sylvestris, Orchis Simia und O. secundiflora.

Unter den dunklen Cypressen, welche urwaldähnlich die hoch aufeinander gethürmten Felsen umstehen, und zu welchen sich herrliche Erdbeerbäume, Arbutus Andrachne, mit korallenroth berindeten Stämmen, lederartigen, glänzenden Blättern und grünlichweissen Blüthenrispen gesellen, stand Lotus peregrinus so massenhaft und reichblüthig, dass der Boden ganz gelb von ihm leuchtete; untergeordnet traten daneben auf: Ainsworthia cordata, Ervum gracile, E. Lenticula, Lathyrus saxatilis, Piptatherum coerulescens, Milium vernale, 
Bromus madritensis, Ajuga tridactylites, Specularia falcata, Rodigia commutata, Seriola aetnensis, Crepis Sieberi.

Nicht ohne Mühe sind diese Felspartion zu erklimmen. Von ihren Zinnen herab bietet sich der entzückende Ausblick nach Norden über den grünen Inselstrand und das dunkelblaue Meer, der, übereinstimmend mit den Aussichten, welche die Nordkette allerorts nach dieser Richtung hin bietet, doch immer wieder ganz überraschend wirkt und immer neue Reize gewährt, so dass das Auge nie müde wird. Auch hier stürzen die Felsen in fast senkrechten Wänden zur Tiefe, herrliche Waldvegetation, vornehmlich Cypressen, Erdbeerbäume, Meerstrandskiefern und Oleander, darunter einzelne uralte, flechtenüberwucherte Pistacien ( $P$. vera?), noch ohne Laub, reichlich aber mit gelbrothen Blüthenrispen geschmückt, zieht sich die Schluchten herauf, - auch hier ein Gürtel grüner, wellenförmig sich hinziehender Vorberge, der vielbuchtige Strand und das wundervolle Meer, - Alles aber anders gruppirt und ganz unfassbar in seiner Schönheit. Wie duftige Wolkengebilde tauchen am fernen Horizont die Berge Anatoliens aus den Fluthen, - durch Spiegelung der Luft zuweilen in umbrandete Waldinseln verwandelt und trügerisch nahe gerückt.

Auf beträchtlichem Umwege gelangten wir hinab unter die Felswände in ein Gebiet, dessen urwüchsige Wildniss durch keines Menschen Fuss entweiht schien. Cypressen- und Erdbeerbäume bilden oft undurchdringliche Dickichte; gewaltige Felsblöcke liegen am Hange umher; flechtenüberwucherte umgestürzte Baumstämme modern am blumigen Boden. Lecokia cretica bildet allerwärts kleine Dickichte für sich oder in Gesellschaft der schönen Scrophularia peregrina mit dem freudiggrünen Laube und den dunkelpurpurrothen Blüthchen. Lose mit Steinen bedeckte Plätze nimmt das üppige, kupferroth leuchtende Geranium lucidum fast für sich allein in Anspruch, selten nur mischt sich Geranium Robertianum dazwischen. Die zarte Saxifraga hederacea bildet gemeinschaftlich mit Veronica cymbalaria, Galium Aparine, Fumaria macrocarpa und Rubia brachypoda so dichte Gewirre am Boden, dass es schwierig ist, einzelne Individuen aus dieser Gesellschaft loszulösen. Nicht minder massenhaft treten Rodigia commutata, Lagoseris bifida, Vaillantía muralis, Galium tricorne, $G$. setaceum, Valerianella truncata, $V$. coronata, V. echinata, Thelygonum Cynocrambe, Euphorbia Helioscopia, Asperugo procumbens etc. auf. Der saftstrotzende Umbilicus pendulinus mit handtellergrossen, kreisrunden Blättern und an die zwei Fuss hohen Blüthenschäften rückt ebenfalls zu kleinen Gruppen von ganz wunderbarem Aussehen, zusammen. - Die umherliegenden Felsblöcke sind mit bunten Moospolstern bedeckt; eine liebliche Flora schmückt sie noch ausserdern, meist kleine, zierliche Pflänzchen und Zwerge aller Genannten. Vor Allen ist Arenaria oxypetala zu nennen, die Millionen kleiner, weisser Blüthensternchen umherstreut, ihr schliesst sich in massenhafter Verbreitung Alsine tenuifolia an. Die violetten Blüthchen der reizenden Arabis verna und die pfirsichblüth-

oesterx. botan. Zeitschrift. 6 . Heft 1882 . 
rothen der Malcolmia chia lenchten dazwischen. Myosotis hispida, Saxifraga lridactylites, Specularia falcata, Thlaspi perfoliatum, Ervum gracile, Lathyrus saxatilis, Ononis reclinata, kleine Gräser, wie Festuca ciliata, Poa rigida, Vulpia sciuroides, Cynosurus elegans, Aira capillaris, Melica saxatilis, Psilurus nardoides u. a. Sie alle tragen das ihrige zur Lieblichkeit dieser Gruppen bei. Als unvergleichliche Zierde will ich noch besonders die zwergigen, reichblüthigen Pflänzchen der Scrophularia peregrina nennen.

Diese Liliputflora nimmt auch von allen ihr zusagenden Stellen der Felswände Besitz. Den schönsten Schmuck aber erhalten letztere durch dio prächtige Arabis albida var. Billardieri, die in reichblïthigen Büschen an ihnen niederbängt. Farnkräuter, Polypodium vulgare und Aspidium Filix mas wuchern üppig aus den Spalten; krüppelhafte Cypressenbüsche und gedrungene Lorbeersträucher finden sogar hier und da noch ein bescheidenes Plätzchen an ihnen anf schmalen Simsen und in den Klüften.

Aller Orten aber, an und auf den Felsen, um die modernden Baumstämme unter dem dunklen, duftigen Nadelgrïn leuchten die lieblichen, zartrosafarbigen Cyclamen-Blüthen.

Die nächste Excursion fübrte uns zu den Ruinen des Schlosses von Kantara, welche anderthalb Stunden östlich vom Kloster auf einem schroff über den Gebirgszug sich erhebenden Felsenkegel gelegen sind. Den Südhang des Bergkammes entlang nahmen wir unseren Weg, vorüber an dem schönen Cypressenhaine. Ein Wäldchen prächtiger Erdbeerbäume schliesst sich an letzteren an; die ziemlich gleichmässig 15-20' hohen, mannigfaltig mit Anschwellungen versehenen Stämme erscheinen in ihrer glatten, rothen Rinde wie aus Kupfer gegossen; die noch etwas luftigen Kronen trugen fast ebenso viel Blüthenrispen wie Blätter. Weiterhin bedeckt die steinigen Hänge das dichte Gestrüpp von Salbei und Cistrosen mit der ihm untergeordnoten niedrigen Flora; oft zeigte sich hier der sonderbare, gelb und orangeroth gefärbte Schmarotzer der Cystensträucher: Cytinus Hypocystis. Stellenweise dominirt die dornige Genista acanthoclada, deren Stämme und Aeste von den ansehnlichen Gehäusen einer Sehnecke förmlich incrustirt werden; wir nahmen eine Masse dieser Thiore als hochwillkommene Bereicherung unserer ärmlich bestellten Küche mit. - Selten sind etwas feuchtere Stellen auf dieser Seite des Gebirges, hohe Oleander- und Myrthengebüsche machen sie schon von weitem kenntlich. Unter Binsen und Riedgräsern hat sich hier gewöhnlich Inula viscosa angesiedelt, die sich zu dieser Jahreszeit abor nur unschön durch ihre verdorrten, über mannshohen, mit alten Blättern noch reichlich besetzten Stauden präsentirt. Auch fehlt es nicht an schroffen Felswänden, längs denen sich in der Regel hohes, fast undurchdringliches Gebüsch erstreckt, überaus reichlich mit Dornen und Nesseln verwehrt.

Wir liessen uns einmal verleiten, diese Wildniss zu durchdringen, um uns über die Pflanze Gewissheit zu verschaffen, welche diese Felswände so schön mit leuchtend gelben Blüthen schmückte. 
Hyoscyamus aureus war der Verführer. Hunderte von Wildtanben und Dohlen, zahlreiche Kohlraben (Corvus corax), kleine Falken und mehrere Geier flüchteten schreiend bei unserem Annahen und dem Krachen der Aeste aus den Felsenklüften, theils sich in höhere Regionen schwingend, theils ängstlichen Fluges ab und zu flatternd. Die Excremente dieser Vögel, von denen die Felsen stellenweise weiss betüncht erschienen, haben den Boden dicht unterm Gestein reichlich gedüngt, und namentlich die Nessel (Urtica pilulifera) bildet hier so enorm hohe Dickichte, dass man befürchten muss, bis über den Hals einzusinken. Mit zerrissenen Kleidern, arg zerzausten Haaren und blutig geritzten Händen gelangten wir nach geraumer Weile mühselig wieder hinaus unter die friedlichen Cistrosen, und wir hatten mit dieser einen Partie an derartigen Oertlichkeiten genug für alle Zeit.

Das in seiner oberen Region ziemlich steil abfallende Gebirge hat oft schluchtenartige Einschnitte, die sich nach der Tiefe hinab verbreitern und zu bergetrennenden Thälern werden. Solche Stellen sind sehr schlecht zu passiren, denn meist bedeckt sie eine lockere Mergelschicht, die in ihrer abschüssigen Lage beim Auftreten leicht ins Rutschen geräth. - Die letzte Wegstrecke legten wir auf der Höhe des Gebirgskammes zurück. Castello Kantara präsentirte sich in seiner gewaltigsten Position; der Anblick dieser Ruine machte uns erstaunen. Aus schwindelnden Tiefen ragt vom grünen Nordstrand her der Felscoloss empor, welcher auf seinen gewaltigen Zinnen die stolz über $\mathrm{z}$ wei Meeren thronende Veste trägt. Unübersehbar nach Nord und nach Süd fluthen blendend die glänzenden Wogenmassen, zwischen welche der cypressenbewachsene Carpass trennend seinen gewaltigen Gebirgsstock stellt. - Die Burg in ihrer kühnen Lage erinnert an Castello della regina; die Ueberreste theilweise bis unter die Dächer erhalten, harmoniren in düsteren Farbentönen und gigantischer Bauart derart mit den Felsensockeln, dass sie durch Naturgewalten aus diesen herausgewachsen erscheinen; erst bei genauerem Hinschauen wird man sich klar, wo die Natur aufhört und das Werk der Menschenhand beginnt.

Die durch ihre Grossartigkeit erdrückend auf den Beschauer wirkende wilde Scenerie muthet aber zugleich auch überaus lieblich durch die sie in prangender Fülle mit Blüthen und mannigfaltigstem Grün umschmiegende Pflanzenwelt an. Die decorative Schönheit namentlich zweier Pflanzen, Cyclamen latifolium und Ferula Anatrichis kommt hier zu vollster Geltung; in unglaublicher Ueppigkeit wuchert das erstere auf allen Felsenrampen, herrliche Festons bildend, während die Riesen-Umbellate mit ihrem feinzerschnittenen Laube und den grossen, goldgelben Blüthenschirmen als entzückende Solitärpflanze auf den breiteren Vorsprüngen der Felswände prangt.

(Fortsetzung folgt.) 